\title{
Mast cell activation and response to tolterodine in the rat urinary bladder in a chronic model of intravesical protamine sulfate and bacterial endotoxin-induced cystitis
}

\author{
BO-HWA CHOI ${ }^{1}$, LONG-HU JIN ${ }^{2}$, KHAE-HAWN KIM ${ }^{3}$, JEE-YOUNG HAN ${ }^{4}$, \\ JU-HEE KANG ${ }^{1}$, SANG-MIN YOON ${ }^{5}$, CHANG-SHIN PARK ${ }^{1}$ and TACK LEE ${ }^{5}$
}

\author{
${ }^{1}$ Department of Pharmacology, Inha University College of Medicine by BK 21 Project, Incheon 400-711, South Korea; \\ ${ }^{2}$ Department of Urology, Shanghai Tenth People's Hospital, Tongji University School of Medicine, Shanghai 200072, \\ P.R. China; ${ }^{3}$ Department of Urology, Gachon University Gil Medical Center, Incheon 405-760, South Korea; \\ Departments of ${ }^{4}$ Pathology and ${ }^{5}$ Urology, Inha University College of Medicine by BK 21 Project, \\ Incheon 400-711, South Korea
}

Received July 21, 2013; Accepted March 27, 2014

DOI: $10.3892 / \mathrm{mmr} .2014 .2262$

\begin{abstract}
The aim of the present study was to use an animal model of interstitial cystitis (IC) in order to investigate the histology and function of the bladder, with a particular focus on mast cell degranulation and response to detrusor overactivity (DO) to tolterodine. A total of 18 female Sprague-Dawley rats were used. In 12 rats, lipopolysaccharide (LPS) was intravesically instilled following the induction of IC by protamine sulfate (PS) and six rats were subjected to sham instillations. Following 1 month, cystometry was performed. The effects of tolterodine were tested in half of the animals with IC. All rats in the IC group demonstrated DO during the filling phase and no significant changes in the frequency or pressure compared with that following tolterodine injection were identified. Histological examination revealed a significant increase in the total number of infiltrated mast cells in IC rats compared with that in the sham rats $(\mathrm{P}<0.05)$. Degranulated mast cells were
\end{abstract}

Correspondence to: Dr Tack Lee, Department of Urology or Dr Chang-Shin Park, Department of Pharmacology, Inha University College of Medicine, 5th Floor Jung-Suk Building, 7-241, 3-Ga, Shinheung-Dong, Jung-Gu, Incheon 400-712, South Korea

E-mail: lee.tack33@gmail.com

E-mail: parkshin@inha.ac.kr

Abbreviations: $\mathrm{BC}, \mathrm{MV}+\mathrm{RV}$; $\mathrm{BP}$, basal pressure; DO, detrusor overactivity; GAG, glycosaminoglycan; IAP, intraabdominal pressures; IC, interstitial cystitis; IVP, intravesical pressure; LPS, lipopolysaccharide; MI, intervals between micturition contractions; MP, maximum bladder pressure during the micturition cycle; MV, micturition volume; $\mathrm{OAB}$, overactive bladder; PS, protamine sulfate; $\mathrm{RV}$, remaining urine after voiding; SEM, standard error of the mean; TP, threshold pressure

Key words: cystitis, interstitial, urinary bladder, mast cells, cystometry, rat evident in $80 \%$ of rats with IC; however, they were not apparent in the sham rats. Urinary bladder inflammation, similar to that in human IC in terms of degranulated mast cells and bladder function, was induced in rats. The animal model used in the present study provided insight into the pathophysiological mechanisms underlying the ineffectiveness of anticholinergics in patients with overlapping IC and overactive bladder (OAB).

\section{Introduction}

Interstitial cystitis (IC) is a chronic, noninfectious inflammatory disease of the bladder that is characterized by pelvic or perineal pain associated with the bladder, irritative voiding symptoms and cystoscopic or histological features (1). In spite of numerous studies, the etiology and pathogenesis of IC remain to be fully elucidated. A few pieces of crucial evidence from pathological biopsies of the human bladder suggested that mast cells are important in the pathogenesis and pathophysiology of IC $(2,3)$. However, there currently is a lack of appropriate rat models of mastocytosis.

Although IC is regarded as a heterogeneous clinical condition that is diagnosed predominantly in females, the current accepted theory is that toxic substances from urine infiltrate the bladder wall owing to disruption of the glycosaminoglycan (GAG) layer, an important permeability barrier to protect the bladder from urine (4). This defective protection followed by increased urothelial permeability may activate mast cells. Mast cells, in turn, release toxic chemical mediators preformed in granules into the surrounding tissue during a process known as degranulation, which results in characteristic degranulated mast cells (2-4). On the basis of this hypothesis, Stein et al (5) developed a novel bladder injury model that mimics IC in which protamine sulfate (PS) and the endotoxin lipopolysaccharide (LPS) are administered intravesically to Sprague-Dawley rats. However, this study mainly focused on the early changes to the injured bladder and did not assess chronic functional changes in awake voiding and degranulation of mast cells in the bladder. Furthermore, Soler et al (6) demonstrated that 
urine has an important role in the development of bladder inflammation in this condition of increased urothelial permeability, which suggests that a chronic bladder injury animal model including urine exposure time to the injured mucosa is required.

Certain patients with an overactive bladder (OAB) and urodynamic detrusor overactivity (DO) demonstrate a positive result on the potassium sensitivity test, which is a characteristic of IC (7). This overlap between OAB and IC remains to be fully elucidated; however, previous studies examining the pathophysiologies of these diseases have provided evidence that the symptoms of patients similarly originate from increased afferent innervations to the lower urinary tract $(8,9)$. Therefore, a good response to anticholinergic medication is expected in patients with IC as compared with patients with OAB. However, previous clinical evidence suggests that numerous patients with IC and DO do not respond to anticholinergic medications (10). The detailed mechanisms associated with this ineffectiveness require investigation in a reliable animal model of IC (11).

Urinary urgency is an essential symptom in the diagnosis of $\mathrm{OAB}$ and is unanimously regarded to correspond with DO during the filling phase according to a human urodynamic study $(12,13)$. A previous study by our group demonstrated that nonvoiding contractions interpreted solely on the basis of intravesical pressure (IVP) need to be corrected by the simultaneous changes in intraabdominal pressures (IAP) in animal models of various diseases in order to use those as a substitute parameter for DO in humans $(14,15)$. In the present study, simultaneous measurements of IVP and IAP were used to assess DO.

The aim of the present study was to use an animal model of chronic IC to assess the effect of inflammation on the histology and function of the urinary bladder in rats, with a particular focus on the degranulation of mast cells and DO during the filling phase of awake voiding. The measurements were made four weeks after exposure to LPS (Sigma, St. Louis, MO, USA) following destruction of the GAG layer by PS (Sigma). In addition, the effects of tolterodine (Sigma) on DO were assessed in this chronic IC model.

\section{Materials and methods}

Animals and study design. A total of 18 female Sprague-Dawley rats (250-300 g; Orient Bio Inc., Gyeonggi-do, South Korea) were used in the present study. In 12 rats, LPS was intravesically instilled following the induction of IC by the administration of PS. In six rats, the intravesical instillation of saline was used as a sham treatment. Cystometrograms were obtained in all unanesthetized, unrestrained rats in metabolic cages, 1 month following intravesical instillation of LPS or saline. Following the completion of the experiments, 15 rats survived, including five out of the six sham-operated controls and 10 out of 12 animals in the IC group. From the IC group, six rats were sacrificed immediately following cystometry and the bladders were removed and examined histologically for mast cell and inflammatory changes. In the remaining rats from the IC group, cystometrograms were obtained following treatment with tolterodine.
Surgical procedures. All experimental animal procedures were performed in accordance with the Guide for the Care and Use of Laboratory Animals of the National Institutes of Health (Bethesda, MD, USA) and were approved by the the INHA Institutional Animal Care and Use Committee at the Inha University Medical School (Incheon, South Korea; approval ID: INHA 100507-57). The rats were maintained under a standard 12-h light/dark cycle and with free access to food pellets and tap water except during the experiments. The rats were anesthetized with ketamine (Ketamine; Yuhan Corp., Seoul, Korea; $75 \mathrm{mg} \mathrm{kg}^{-1}$ intraperitoneally) and xylazine (Rompun; Bayer Korea Corp, Seoul, Korea; $15 \mathrm{mg} \mathrm{kg}^{-1}$ intraperitoneally) for specific procedures.

Induction of cystitis. Cystitis was induced by intravesical instillation of LPS following PS, as described by Stein et al (5) with certain modifications. Through an abdominal incision under anesthesia, a 24-gauge needle with a syringe was inserted into the dome of the bladder. After all the urine was aspirated, $0.5 \mathrm{ml}$ of PS (10 mg ml-1) was instilled into the bladder lumen. Following $20 \mathrm{~min}$, the bladder was emptied, washed with phosphate-buffered saline (PBS) and then given a second treatment with $0.5 \mathrm{ml}$ of LPS $(750 \mu \mathrm{g} / \mathrm{ml})$ for $20 \mathrm{~min}$. For the sham group, normal saline of the same volume was instilled.

Procedures for intravesical, intraabdominal and intravenous catheter implantation. Simultaneous catheterizations for IVP and IAP recordings were performed three days prior to cystometry, as described previously (11,13-15). Briefly, following the induction of anesthesia, a polyethylene catheter (PE-50; Becton-Dickinson, Parsippany, NJ, USA) with a cuff was implanted into the dome of the bladder through an abdominal incision. To record IAP, an abdominal balloon (Latex; Daewoo Medical, Incheon, Korea) around the cuff of a catheter tip was placed proximal to the bladder and was tied to another catheter with a silk tie. A polyethylene catheter (PE-50) was heated in warm water, elongated by $\sim 1.5$ times its original length at the tip of the inserting side and filled with heparinized saline $\left(100 \mathrm{IU} \mathrm{ml}^{-1}\right)$. As the bladder catheter was implanted, the elongated catheter was inserted into the femoral vein in six out of the 12 rats in the IC group in the same session. These catheters were then tunneled through the subcutaneous space, exited through the back of the animal and were anchored to the skin of the back. Following surgery, each rat was caged individually and maintained in the same manner.

Functional evaluation. Cystometrograms were performed on unanesthetized, unrestrained rats in metabolic cages. The indwelling catheter to the bladder was connected to a two-way valve that was connected via a T-tube to a pressure transducer (Research Grade Blood Pressure Transducer; Harvard Apparatus, Holliston, MA, USA) and a microinjection pump (PHD22/2000 pump; Harvard Apparatus). Another indwelling catheter connected to a fluid-filled abdominal balloon was connected to another pressure transducer to record the IAP. The micturition volumes were recorded continuously by means of a fluid collector connected to a force displacement transducer (Research Grade Isometric Transducer; Harvard 
Table I. Cystometric parameters in conscious, unrestrained Sprague-Dawley rats in the sham and IC groups.

\begin{tabular}{lcccccccc}
\hline Group & $\begin{array}{c}\mathrm{BP}[\mathrm{DP} \\
\left.\left(\mathrm{cm} \mathrm{H}_{2} \mathrm{O}\right)\right]\end{array}$ & $\begin{array}{c}\mathrm{TP}[\mathrm{DP} \\
\left.\left(\mathrm{cm} \mathrm{H}_{2} \mathrm{O}\right)\right]\end{array}$ & $\begin{array}{c}\mathrm{MP}[\mathrm{DP} \\
\left.\left(\mathrm{cm} \mathrm{H}_{2} \mathrm{O}\right)\right]\end{array}$ & $\mathrm{BC}(\mathrm{ml})$ & $\mathrm{MV}(\mathrm{ml})$ & $\mathrm{RV}(\mathrm{ml})$ & $\mathrm{MI}\left(\mathrm{min}^{-1}\right)$ & $\begin{array}{c}\text { DO positive, } \\
\mathrm{n}(\%)\end{array}$ \\
\hline Sham $(\mathrm{n}=5)$ & $6.60 \pm 1.48$ & $18.98 \pm 3.22$ & $53.56 \pm 5.72$ & $1.24 \pm 0.21$ & $1.24 \pm 0.21$ & $0.00 \pm 0.00$ & $8.08 \pm 1.38$ & $0(0 \%)$ \\
IC $(\mathrm{n}=11)$ & $6.80 \pm 0.59$ & $18.15 \pm 0.59$ & $57.27 \pm 3.42$ & $1.45 \pm 0.15$ & $1.45 \pm 0.15$ & $0.00 \pm 0.00$ & $8.73 \pm 0.73$ & $11(100 \%)$ \\
\hline
\end{tabular}

IC, interstitial cystitis; Pr, pressure; BP, basal pressure; TP, threshold pressure; MP, micturition pressure; BC, bladder capacity; MV, micturition volume; RV, residual volume; MI, micturition interval; DO, detrusor overactivity; DP, detrusor pressure. Results are expressed as the mean \pm standard error.

Apparatus). Room-temperature saline was infused into the bladder at a rate of $10 \mathrm{ml} \mathrm{h}^{-1}$. IVP, IAP and micturition volumes were recorded continuously using Acq Knowledge 3.8.1 software and an MP150 data acquisition system (Biopac Systems, Goleta, CA, USA) at a sampling rate of $50 \mathrm{~Hz}$. The mean values from three reproducible micturition cycles were used for evaluation. IAP was defined as the recorded balloon pressure subtracted by the lowest balloon pressure in each voiding cycle (zeroing). The detrusor pressure was defined as the IVP minus IAP. The increase in intravesical pressure during the filling phase was defined as increments of IVP that exceeded $2 \mathrm{~cm} \mathrm{H}_{2} \mathrm{O}$ from baseline, which was interpreted as DO if occurring without simultaneous similar changes in IAP or abdominal straining if occurring with simultaneous similar changes in IAP.

Investigation of cystometric parameters. Pressure- and volume-associated parameters consisted of the lowest bladder pressure during filling (BP), bladder pressure immediately prior to micturition (TP), maximum bladder pressure during the micturition cycle (MP), volume of expelled urine (MV), remaining urine following voiding $(\mathrm{RV}), \mathrm{MV}+\mathrm{RV}(\mathrm{BC})$ and intervals between micturition contractions (MI).

DO-associated parameters during the filling phase. These consisted of the time of the filling phase (interval from the initiation of infusion through the tube and the point immediately prior to the initiation of micturition), frequency of abdominal straining per minute, frequency of DO per minute and increased amplitude from base to peak of the DO spike as IVP. These frequencies were calculated on the basis of the time of the filling phase.

Administration of the drug. During cystometry, room temperature saline was infused into the bladder at a rate of $10 \mathrm{ml} \mathrm{h}^{-1}$. The micturitions during intravesical saline infusion served as baseline values. After $0.2 \mathrm{ml}$ tolterodine solution $\left(0.3 \mathrm{mg} \mathrm{kg}^{-1}\right)$ was injected intravenously, there was a $30 \mathrm{~min}$ observation period. Following cystometry, the animals were sacrificed by cervical dislocation. The bladder and the urethra were removed en bloc and separated at the level of the bladder neck, and the bladder was weighed.

Histological evaluation. As previously described, the bladder was excised, split longitudinally and fixed in $10 \%$ buffered formaldehyde and embedded in paraffin. Thin sections
( $4 \mu \mathrm{m})$ of the bladder were cut and stained with $1 \%$ toluidine blue (Merck, Darmstadt, Germany) to assess inflammatory changes as well as the number of total and degranulated mast cells. Slides were examined with an Olympus BX51 light microscope (Olympus, Tokyo, Japan) and images were captured with an Olympus PM10SP photographic system. For quantification, mast cells in the mucosa and muscularis were counted and averaged from five randomly selected microscopic visual fields at x200 magnification under an optical microscope (Olympus).

Statistical analysis. All results were analyzed using SPSS 20.0 (SPSS Inc., Chicago, IL, USA). The results are presented as the mean values \pm standard error of the mean. Normal distributions were confirmed by the Shapiro-Wilk W-test. Statistical significance was determined by unpaired t-tests to detect differences in urodynamic parameters and histological data between the sham and IC groups. Paired t-tests were used to compare these parameters prior to and following drug administration in half of the IC group. For multiple comparisons, one-way analysis of variance with Tukey's test was used to detect differences. $\mathrm{P}<0.05$ was considered to indicate a statistically significant difference. All calculations were made on the basis of $\mathrm{n}$, which denoted the number of animals.

\section{Results}

Body weight, bladder weight and ratio. No significant difference in the body weight between the sham group $(272.0 \pm 5.6 \mathrm{~g})$ and the IC group $(275.0 \pm 5.2 \mathrm{~g})$ was identified four weeks following intravesical instillations of PS and LPS or saline. The bladder weight in the IC group $(0.14 \pm 0.00 \mathrm{~g})$ did not differ significantly from that of the sham group $(0.14 \pm 0.01 \mathrm{~g})$ and no significant differences between the groups were identified when the bladder weight was normalized to body weight (data not shown).

Comparison of the urodynamic parameters between the sham and IC groups. Rats with ICs did not differ significantly from the sham rats in any pressure- or volume-asssociated parameters, including BP, TP, MP, BC, MV, RV and MI (Table I). However, DO during the filling phase was shown in all rats in the IC group (100\%), but was not apparent in the sham group $(0 \%$; Table I). The average frequency and pressure of DO in the IC group was $1.56 \pm 0.41 \mathrm{~min}^{-1}$ and $2.61 \pm 0.66 \mathrm{~cm} \mathrm{H}_{2} \mathrm{O}$, respectively (data not shown). 
Table II. Changes in cystometric parameters in conscious, unrestrained Sprague-Dawley rats in the IC group following intravenous injection of tolterodine.

\begin{tabular}{|c|c|c|c|c|c|c|c|c|}
\hline $\mathrm{IC}$ rats & $\begin{array}{c}\mathrm{BP}[\mathrm{DP} \\
\left.\left(\mathrm{cm} \mathrm{H} \mathrm{H}_{2} \mathrm{O}\right)\right]\end{array}$ & $\begin{array}{c}\text { TP [DP } \\
\left.\left(\mathrm{cm} \mathrm{H} \mathrm{H}_{2} \mathrm{O}\right)\right]\end{array}$ & 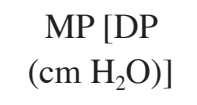 & $\mathrm{BC}(\mathrm{ml})$ & $\mathrm{MV}(\mathrm{ml})$ & $\mathrm{RV}(\mathrm{ml})$ & $\mathrm{MI}\left(\min ^{-1}\right)$ & $\begin{array}{l}\text { O positive, } \\
\text { n }(\%)\end{array}$ \\
\hline Control (n=4) & $6.40 \pm 0.86$ & $16.08 \pm 1.37$ & $51.08 \pm 6.36$ & $1.62 \pm 0.28$ & $1.56 \pm 0.24$ & $0.06 \pm 0.06$ & $9.52 \pm 1.82$ & $4(100 \%)$ \\
\hline Tolterodine IV $(n=4)$ & $6.60 \pm 0.65$ & $11.78 \pm 1.04$ & $18.13 \pm 5.90^{*}$ & $1.63 \pm 0.22$ & $1.56 \pm 0.17$ & $0.07 \pm 0.07$ & $10.24 \pm 1.47$ & $4(100 \%)$ \\
\hline
\end{tabular}

IC, interstitial cystitis; Pr, pressure; BP, basal pressure; TP, threshold pressure; MP, micturition pressure; BC, bladder capacity; MV, micturition volume; RV, residual volume; MI, micturition interval; DO, detrusor overactivity; DP, detrusor pressure. Results are expressed as the mean \pm standard error. ${ }^{*} \mathrm{P}<0.05$ (paired Student's t-test) vs. control.

A
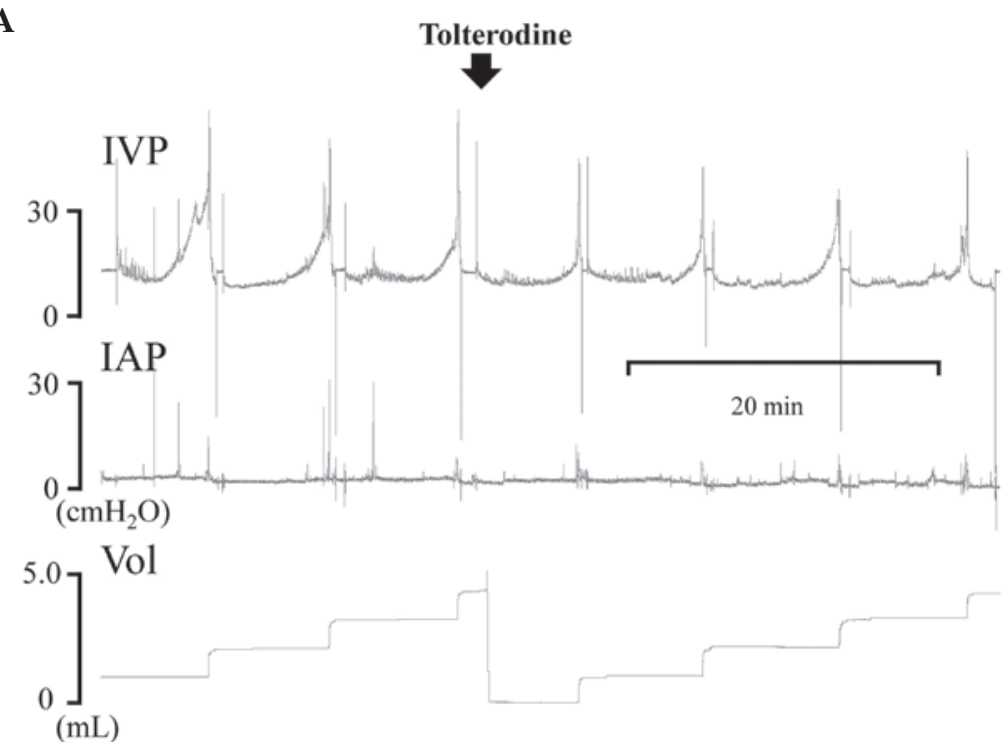

B

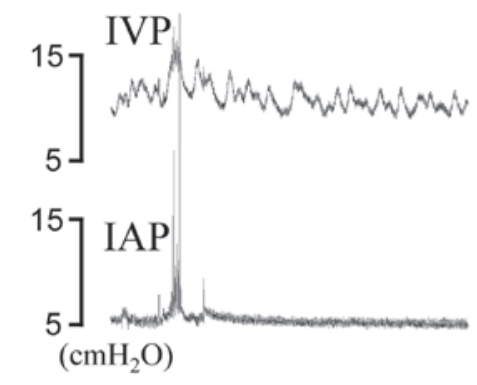

D

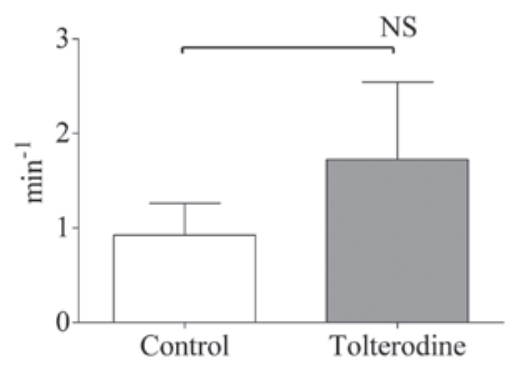

C

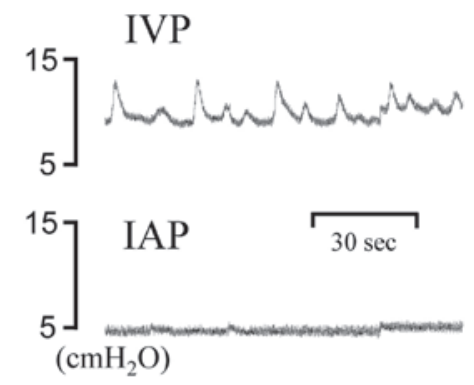

E

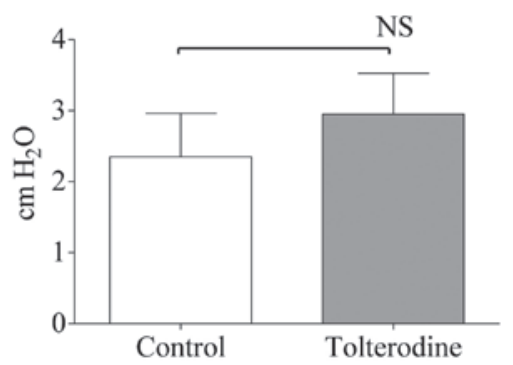

Figure 1. (A) Representative cystometrogram from one rat demonstrating IVP, IAP and Vol prior to and following intravenous injection of 0.3 mg $\mathrm{kg}^{-1}$ of tolterodine 1 month following the induction of IC by intravesical administration of LPS following PS. (B) During the filling phase, the fluctuations in IVP recordings were not interpretated as DO, if occurring with simultaneous similar changes in IAP. The black arrow indicates the abdominal straining. (C) IVP recordings were interpretated as DO if occurring without simultaneous changes in IAP. Prior to the injection, all IC rats showed DO. Following the injection, no significant changes in (D) frequency or (E) pressure of DO were present. NS, not significant. IVP, intravesical pressure; IAP, intraabdominal pressure; Vol, micturition volume; IC, interstitial cystitis; LPS, lipopolysaccharide; PS, protamine sulfate; DO, detrusor overactivity. 
A

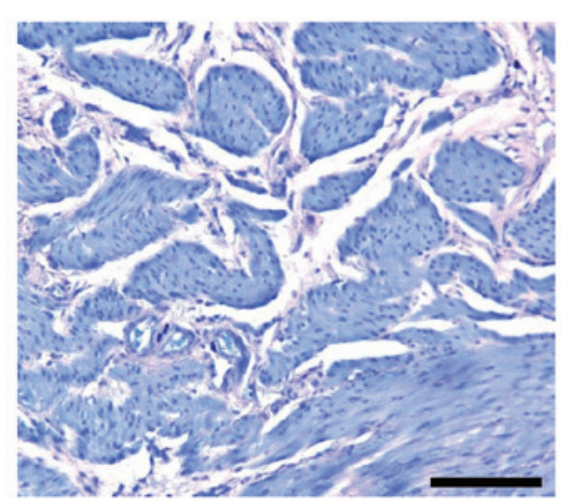

C

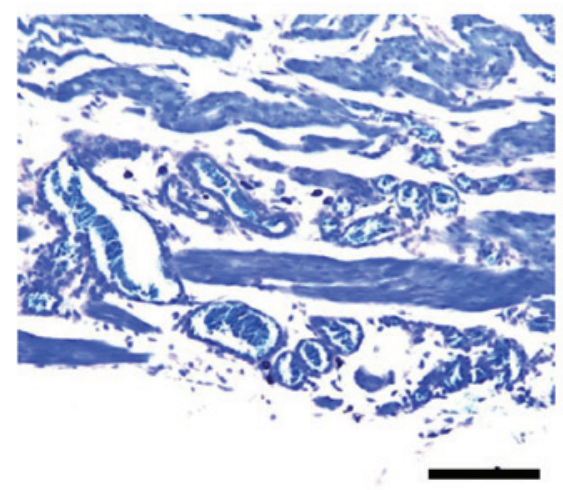

E

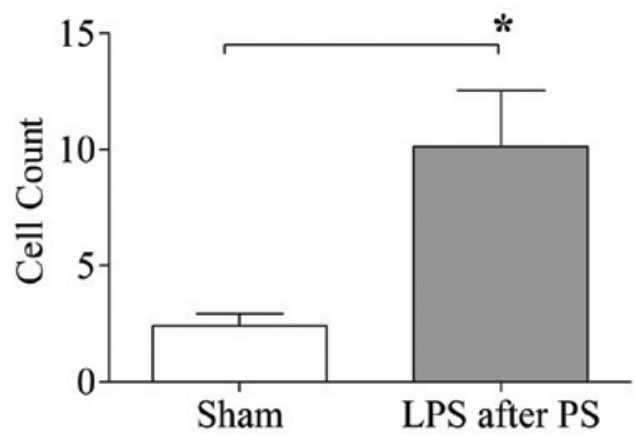

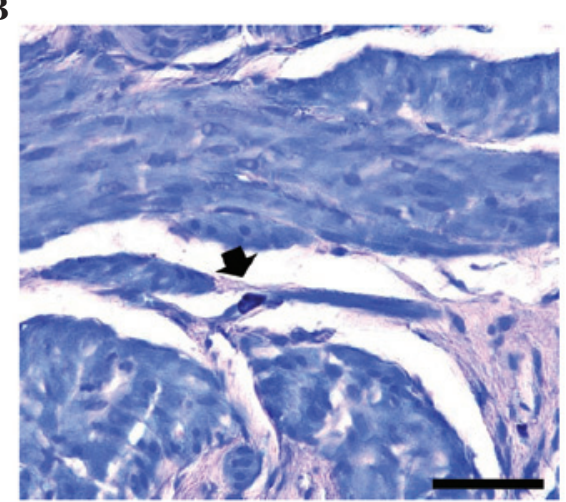

D

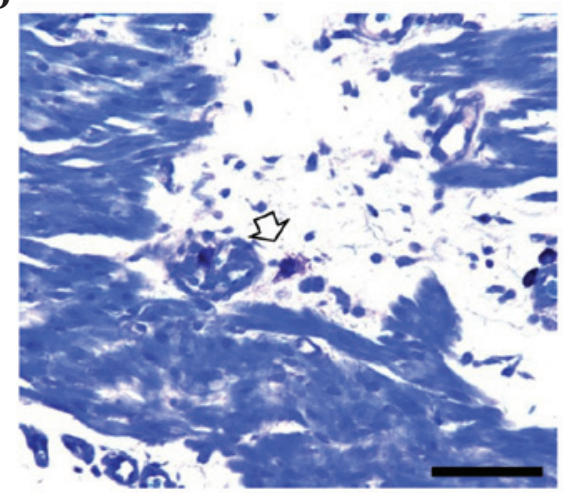

Q Granulated MC only

$\square$ Degranulated MC found

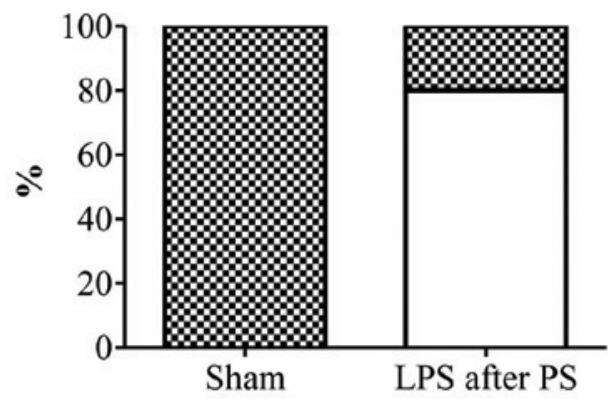

Figure 2. Photomicrographs of the rat bladder following toluidine blue staining demonstrating granulated (black arrow) and degranulated (white arrow) mast cells. (A) Histological appearance of the urinary bladder wall with rare mast cells in sham rats (magnification, x200). (B) These cells were all granulated (magnification, x400). (C) Rats demonstrated an increased number of mast cells 1 month following intravesical instillation of LPS following PS (magnification, x200). (D) Characteristic degranulated cells were present in $80 \%$ of these rats (magnification, $\mathrm{x} 400)$. (E) Quantification of the cell count ( $\mathrm{P}<0.05)$. (F) Quantification of granulated and degranulated cells. Bars, $100 \mu \mathrm{m}$ in a and c; Bars, $50 \mu \mathrm{m}$ in b and d. LPS, lipopolysaccharide; PS, protamine sulfate.

Changes in the urodynamic parameters following the intravenous injection of an anticholinergic drugs in rats during IC. Compared with prior to tolterodine injection, no significant differences in any pressure-associated parameters, including $\mathrm{BP}$ and TP with the exception of MP, were identified following tolterodine treatment in the IC group (Table II). Following treatment, the MP decreased significantly in IC rats from 51.08 \pm 6.36 to $18.13 \pm 5.90 \mathrm{~cm} \mathrm{H}_{2} \mathrm{O}(\mathrm{P}<0.05$; Fig. 1A). No significant differences in any volume-associated parameters, including $\mathrm{BC}, \mathrm{MV}$, $\mathrm{RV}$ and MI, were identified following tolterodine treatment (Table II). Additionally, there were no significant changes in the frequency or pressure of DO during the filling phase prior to and following the medication (Table II; Fig. 1B-E).
Comparison of the histological findings between the sham and IC groups. In total, five rats formed the IC group as one rat was excluded owing to a technical problem. In the sham rats, the urothelium, lamina propria and muscularis propria appeared normal. The rats treated with LPS following PS had a thickened bladder wall and focally atrophied uroepithelium. Histological examination further revealed a significant increase in the total numbers of infiltrated mast cells in the IC rats compared with the sham rats $(\mathrm{P}<0.05$; Fig. 2$)$. Four out of the five treated rats demonstrated degranulated mast cells $(80 \%)$, whereas the sham rats did not (Fig. 2). However, among the total counted mast cells, only $14.2 \%$ were degranulated and $85.8 \%$ were granulated mast cells (data not shown). 


\section{Discussion}

A central observation of the present study was that chronic IC with similar characteristics to the human disease was induced in Sprague-Dawley rats by intravesical instillations of LPS following PS. All rats in this chronic model except one exhibited degranulated mast cells, which was crucial evidence for the successful establishment of a model resembling the human disease. None of the sham rats exhibited degranulated mast cells. PS was used to destroy the GAG layer, which protects the bladder from the adherence of bacteria, to facilitate the action of LPS. Furthermore, irritation by urine over four weeks appeared to have an important role in this model. Although the urodynamic results in these model rats did not match up to all the characteristic features of IC in humans, all rats demonstrated DO during the filling phase, which is a characteristic of OAB.

The pathophysiological mechanisms that have been implicated in IC include uroepithelial dysfunction, mast cell activation and neurogenic inflammation $(2,3,8)$. Among these, the most important is mast cell activation, which can be precipitated by diverse nonimmunological stimulants, including bacteria, chemicals, kinins, neuropeptides and acetylcholine $(2,3,16)$. Although IC is not an infectious disease, LPS, an endotoxin from $E$. coli, is commonly used as an acute precipitating factor in animal models of IC (17). In the normal urinary bladder, however, the GAG layer prevents the adherence of bacteria by covering possible receptor sites $(4,18)$. Thus, intravesical instillation of PS, a highly alkaline polycationic peptide, is used to destroy this GAG layer on urothelial cells prior to the instillation of LPS (19). Until recently, the majority of studies have focused on the acute effects of this dual treatment on the bladder in animal models and few studies have investigated the chronic effects on awake voiding and mast cell degranulation. In the model used in the present study, the injured uroepithelium was further exposed to urine as a chronic irritating stimulant for 1 month, which is expected to be similar to the conditions in patients with IC.

Mast cells are essential in the initiation and propagation of inflammation, leading to numerous symptoms of acute and chronic inflammatory diseases $(3,16,20)$. They function as immune sentinels in almost all vascularized tissues or organs and co-ordinate all the steps of the inflammatory response, with positive and negative actions in the course of numerous diseases, including the defense against microbial infection $(16,20)$. They store proinflammatory mediators, neurotrophic factors and immunoregulatory cytokines in their vesicles and discharge their large population of secretary granules outside the cell into the tissue by stimulating factors. These mediators are important in the immediate and late phase inflammatory reactions. When chronically stimulated, mast cells exhibit characteristic features of degranulated mast cells, also known as phantom cells $(3,21)$. Furthermore, they are associated with neuropathic pain, a characteristic of IC, mediated by neurotrophins, including nerve growth factor (22). The exact etiology and pathophysiology of IC remain to be fully elucidated; however, mast cells are known to be important in IC pathogenesis $(2,23)$. Furthermore, the importance of degranulated mast cells in IC was demonstrated by crucial pathological evidence from numerous human studies $(2,3)$. The present study demonstrated that $80 \%$ of IC model rats exhibited degranulated mast cells, with a significant increase in the total number of mast cells compared with that in the sham rats. However, among the total mast cells, only $14.2 \%$ were degranulated, which suggests that a longer duration of IC is necessary.

The symptoms of IC and OAB overlap considerably, with numerous patients with IC demonstrating urgency (7). By the definition of the International Continence Society (24), IC can be included, in part, in the category of OAB. However, according to the National Institute of Diabetes and Digestive and Kidney Diseases database study, which was the first major prospective trial on IC, none of the urodynamic parameters add enough diagnostic evidence to characterize the voiding behavior of patients with IC as OAB (25), possibly due to the disease heterogeneity. Other population-based studies on IC reported a prevalence of urodynamically verified DO ranging from $14-30 \%(26,27)$. The animal model used in the present study similarly demonstrated no significantly different urodynamic parameters compared with those in the controls; however, all rats demonstrated DO. These findings are similar to those of a previous rat model of IC induced by intravesical administration of $\mathrm{HCl}$ (17). In the present study, rats with IC and DO demonstrated no response in DO parameters to an anticholinergic drug, which differs from the findings of other OAB animal models, including the models of bladder outlet obstruction or intravesical instillation of prostaglandin E2 $(11,28)$. It is suggested that the dissimilar effects of anticholinergic drugs may be associated with the complex cellular and molecular mechanisms in the bladder, which remain to be elucidated.

In conclusion, an improved understanding of the disease heterogeneity through the use of an animal model of chronic IC, which is similar to the human disease condition in view of degranulated mast cells and bladder function, may provide major novel insights into unidentified pathophysiological mechanisms, including those underlying the dissimilar effects of anticholinergic drugs on the different diseases. In addition it may also lead to further progress in the detection and treatment of this complex disease.

\section{Acknowledgements}

This study was supported by Inha University Research grant and Astellas Research grant (ARK-VC-2013-02). The funders had no role in the design, data collection and analysis, decision to publish or preparation of the manuscript.

\section{References}

1. Gillenwater JY and Wein AJ: Summary of the National Institute of Arthritis, Diabetes, Digestive and Kidney Diseases Workshop on Interstitial Cystitis, National Institutes of Health, Bethesda, Maryland, August 28-29, 1987. J Urol 140: 203-206, 1988.

2. Theoharides TC, Kempuraj D and Sant GR: Mast cell involvement in interstitial cystitis: a review of human and experimental evidence. Urology 57: 47-55, 2001.

3. Sant GR and Theoharides TC: The role of the mast cell in interstitial cystitis. Urol Clin North Am 21: 41-53, 1994.

4. Parsons CL: The role of a leaky epithelium and potassium in the generation of bladder symptoms in interstitial cystitis/overactive bladder, urethral syndrome, prostatitis and gynaecological chronic pelvic pain. BJU Int 107: 370-375, 2011.

5. Stein PC, Pham H, Ito T and Parsons CL: Bladder injury model induced in rats by exposure to protamine sulfate followed by bacterial endotoxin. J Urol 155: 1133-1138, 1996. 
6. Soler R, Bruschini H, Freire MP, Alves MT, Srougi M and Ortiz V: Urine is necessary to provoke bladder inflammation in protamine sulfate induced urothelial injury. J Urol 180: 1527-1531, 2008.

7. Chung MK, Butrick CW and Chung CW: The overlap of interstitial cystitis/painful bladder syndrome and overactive bladder. JSLS 14: 83-90, 2010.

8. Yoshimura N and de Groat WC: Increased excitability of afferent neurons innervating rat urinary bladder after chronic bladder inflammation. J Neurosci 19: 4644-4653, 1999.

9. Fowler CJ: Bladder afferents and their role in the overactive bladder. Urology 59: 37-42, 2002.

10. Minaglia S, Ozel B, Bizhang R and Mishell DR Jr: Increased prevalence of interstitial cystitis in women with detrusor overactivity refractory to anticholinergic therapy. Urology 66 : 702-706, 2005

11. Jin LH, Park CS, Shin HY, Yoon SM and Lee T: Dissimilar effects of tolterodine on detrusor overactivity in awake rats with chemical cystitis and partial bladder outlet obstruction. Int Neurourol J 15: 120-126, 2011.

12. Garnett $\mathrm{S}$ and Abrams P. The natural history of the overactive bladder and detrusor overactivity. A review of the evidence regarding the long-term outcome of the overactive bladder. J Urol 169: 843-848, 2003.

13. Lee $\mathrm{T}$ and Yoon SM: The role of intra-abdominal pressure measurement in awake rat cystometry. Int Neurourol J 17: 44-47, 2013.

14. Lee T, Andersson KE, Streng T and Hedlund P: Simultaneous registration of intraabdominal and intravesical pressures during cystometry in conscious rats--effects of bladder outlet obstruction and intravesical PGE2. Neurourol Urodyn 27: 88-95, 2008.

15. Jin LH, Shin HY, Kwon YH, Park CS, Yoon SM and Lee T: Urodynamic findings in an awake chemical cystitis rat model observed by simultaneous registrations of intravesical and intraabdominal pressures. Int Neurourol J 14: 54-60, 2010.

16. Galli SJ: New concepts about the mast cell. N Engl J Med 328: 257-265, 1993

17. Saban MR, Saban R, Hammond TG, Haak-Frendscho M, Steinberg H, Tengowski MW and Bjorling DE: LPS-sensory peptide communication in experimental cystitis. Am J Physiol Renal Physiol 282: F202-F210, 2002.
18. Parsons CL: The role of the urinary epithelium in the pathogenesis of interstitial cystitis/prostatitis/urethritis. Urology 69: 9-16, 2007

19. Parsons CL, Stauffer CW and Schmidt JD: Reversible inactivation of bladder surface glycosaminoglycan antibacterial activity by protamine sulfate. Infect Immun 56: 1341-1343, 1988

20. Yong LC: The mast cell: origin, morphology, distribution, and function. Exp Toxicol Pathol 49: 409-424, 1997.

21. Claman HN, Choi KL, Sujansky W and Vatter AE: Mast cell 'disappearance' in chronic murine graft-vs-host disease (GVHD)-ultrastructural demonstration of 'phantom mast cells'. J Immunol 137: 2009-2013, 1986.

22. Lowe EM, Anand P, Terenghi G, Williams-Chestnut RE, Sinicropi DV and Osborne JL: Increased nerve growth factor levels in the urinary bladder of women with idiopathic sensory urgency and interstitial cystitis. Br J Urol 79: 572-577, 1997.

23. Park CS and Bochner BS: Potential targeting of siglecs, mast cell inhibitory receptors, in interstitial cystitis. Int Neurourol J 15: 61-63, 2011.

24. Abrams P, Cardozo L, Fall M, Griffiths D, Rosier P, Ulmsten U, van Kerrebroeck P, Victor A and Wein A: The standardisation of terminology of lower urinary tract function: report from the Standardisation Sub-committee of the International Continence Society. Neurourol Urodyn 21: 167-178, 2002.

25. Hanno PM, Landis JR, Matthews-Cook Y, Kusek J and Nyberg L Jr: The diagnosis of interstitial cystitis revisited: lessons learned from the National Institutes of Health Interstitial Cystitis Database study. J Urol 161: 553-557, 1999.

26. Nigro DA, Wein AJ, Foy M, Parsons CL, Williams M, Nyberg LM Jr, Landis JR, Cook YL and Simon LJ: Associations among cystoscopic and urodynamic findings for women enrolled in the Interstitial Cystitis Data Base (ICDB) Study. Urology 49: 86-92, 1997.

27. Hanno P, Lin A, Nordling J, Nyberg L, van Ophoven A, Ueda T and Wein A: Bladder Pain Syndrome Committee of the International Consultation on Incontinence. Neurourol Urodyn 29: 191-198, 2010.

28. Oh JH, Lee YS, Jin LH Kwon YH, Park WH and Lee T: Urodynamic effects of propiverine on detrusor overactivity and abdominal straining during voiding in awake rats with intravesical prostaglandin E(2) instillation. Korean J Urol 51: 64-69, 2010. 